\title{
THE PROPERTIES OF MACROCYCLIC COMPLEXES OF RUTHENIUM
}

\author{
Mikailova M.R., Mustafayeva R.E.
}

\section{Azerbaijan State University of Oil and Industry,Baku, Azerbaijan,mehriban-mikayilova@mail.ru}

Ruthenium has many valuable and interesting properties. For many mechanical, electrical and chemical characteristics, it can compete with many metals and even with platinum and gold. However, unlike these metals, ruthenium is very fragile, and therefore it is not possible to make any products from it yet. Apparently, the brittleness and intractability of ruthenium by mechanical treatment are due to the insufficient purity of the samples subjected to the tests. The physical properties of this metal depend very much on the method of production, and no one has yet been able to isolate ruthenium of high purity. Attempts to obtain pure ruthenium by sintering in briquettes, zone melting and other methods did not lead to positive results. For this reason, technically important characteristics such as tensile strength and elongation at break have not yet been accurately determined. Only recently, the melting point of ruthenium is accurately estimated at $2,250^{\circ} \mathrm{C}$, and its boiling point lies somewhere around $4,900^{\circ} \mathrm{C}$. Metal ruthenium very actively sorbs hydrogen. Usually the standard of the hydrogen sorbent is palladium, the cubic centimeter of which absorbs $940 \mathrm{~cm}^{3}$ of hydrogen. The absorption capacity of ruthenium is higher. It sorbs 1,500 volumes of hydrogen [Raevskaya, 1979].

Another important property of ruthenium: at a temperature of $-272.53{ }^{\circ} \mathrm{C}$ it becomes a superconductor. Compact metallic ruthenium is not soluble in alkalis, acids and even in boiling aqua regia, but partially soluble in nitric acid with the addition of strong oxidizing agents - per chlorates or bromates.

Ruthenium can be dissolved in alkaline medium by hypochlorites or in acid medium by electrochemical method. When heated in air, ruthenium begins to partially oxidize. The maximum oxidation rate is observed at $800^{\circ}$ C. Up to a temperature of $1,000^{\circ} \mathrm{C}$, ruthenium is always oxidized only to $\mathrm{RuO}_{2}$, but if it is heated to $1,200^{\circ} \mathrm{C}$ and higher, it begins to convert to volatile $\mathrm{RuO}_{4}$ tetra oxide, exhibiting a higher valence of $8+$.

$\mathrm{RuO}_{4}$ is a very interesting compound. Under normal conditions, these are golden yellow needle-shaped crystals that already melt at $25^{\circ} \mathrm{C}$, turning into a brown-orange liquid with a specific odor similar to that of ozone. When touched with the slightest trace of most organic substances, ruthenium tetroxide momentarily explodes. At the same time, it dissolves well in chloroform and carbon tetrachloride. $\mathrm{RuO}_{4}$ is poisonous: with prolonged inhalation of its vapors, the person begins to feel dizzy, there are attacks of vomiting and suffocation. Some chemists who worked with ruthenium tetroxide developed eczema.

The ability of ruthenium to form tetroxide played an important role in the chemistry of this element. By transferring to volatile $\mathrm{RuO}_{4}$, it is possible to separate ruthenium from other noble and base metals and, after its recovery, obtain the purest ruthenium. In the same way, ruthenium impurities are removed from rhodium, iridium and platinum.

The struggle against radioactive ruthenium is paid much attention to by physicists, chemists, technologists and especially radio chemists of many countries. At the First and Second International Conferences on the Peaceful Uses of Atomic Energy in Geneva, several reports were devoted to this problem.

Ruthenium is the only platinum metal found in living organisms (according to some sources, it is also platinum). It concentrates mainly in the muscle tissue. The highest ruthenium oxide is extremely toxic and, being a strong oxidant, can cause fire of fire hazardous substances.

Ruthenium is a rare and very scattered element. In industry heterogeneous catalysis uses metallic ruthenium and its alloys. Ruthenium is used in the preparation of the most effective catalysts. The present study was carried out in the field of synthetic and structural chemistry of ruthenium complexes with organic nitrogen-containing compounds.

Using ruthenium $\mathrm{Ru}_{3}(\mathrm{CO})_{12}$ as a starting compound and reacting with a phthalocyanine ligand in benzonitrile for 1.5 hours followed by extraction with pyridine, it was possible to obtain in a small yield a ruthenium complex with phthalocyanine containing axially coordinated $\mathrm{CO}$ molecules and pyrolysine, Ru Pc (CO) (py).

Alkyl-substituted ruthenium phthalocyaninates were synthesized from free phthalocyanine with higher yields. $\left(\mathrm{C}_{5} \mathrm{H}_{11} \mathrm{O}\right) \mathrm{SP}_{2} \mathrm{H}_{2}$ and dissolved in boiling 2-ethoxyethanol, a solution of $\mathrm{RuCl}_{3} * 3 \mathrm{H}_{2} \mathrm{O}$ in the same solvent (molar ratio of starting reactants $1: 2$ ) was added and the mixture was boiled for 24 hours.

The cooled solution was poured into $\mathrm{MeOH} / \mathrm{H}_{2} \mathrm{O}$ (3: 1), filtered and dried. The reaction product was then purified by chromatography on neutral alumina $\mathrm{Al}_{2} \mathrm{O}_{3}$ (eluent-chloroform). However, this method failed to obtain a complex without axial ligands: the IR spectrum of the compound synthesized in this way contains a characteristic band at $1900 \mathrm{~cm}-1$, characterizing the valence 
vibrations of - (CO). In the 13C-NMR spectrum of this complex, a signal is observed in the region of $181.06 \mathrm{ppm}$, which also confirms the presence of a $\mathrm{CO}$ molecule in the complex. The yield $\left(\mathrm{C}_{5} \mathrm{H}_{11} \mathrm{O}\right){ }_{8} \mathrm{PcRu}(\mathrm{CO})$ was $30 \%$, for (2-Et-hexO) ${ }_{8} \mathrm{PcRu}(\mathrm{CO}) 25 \%$.

This, on the methods of synthesis and the composition of substituted and unsubstituted ruthenium phthalocyaninates, it can be concluded that the final product of the reaction, regardless of the method of synthesis, almost always contains in its composition a carbonyl group of $\mathrm{CO}$ as an axial ligand.

It was found that the production of pure ruthenium phthalocyaninate, which does not contain any axially coordinated molecules, is possible with the thermal decomposition of complexes of the composition PcRu (CO) $\mathrm{L}$ or $\mathrm{PcRuL}_{2}$. Therefore, many works are devoted to the synthesis and study of ruthenium complexes with phthalocyanines and various axial ligands.
Synthetic methods have been developed and Ru (IV) complex compounds with five types of donor ligands have been prepared: phenanthroline, nicotinic acid amide, imidazole, benzimidazole and their derivatives, and also phthalocyanine.

A new method for the synthesis of rhenium and ruthenium phthalocyanines is proposed, which is distinguished by its availability and high yields. It is established that as a result of synthesis only one form of ruthenium phthalocyanine is formed, which can be attributed to the $\mathrm{PcRuO}$ structure on the basis of physical and chemical studies.

\section{LITERATURE}

1. Raevskaya MV: Physicochemistry of ruthenium and its alloys / MV Raevskaya // Chemistry of ruthenium, rhodium, palladium, osmium, iridium, platinum, per. With the English. M .: 1979. - P. 138 - 140. 